\title{
古北界狗獾食性的生物地理模式: 狗獾是虹蚛专食者四？
}

\author{
李峰 ${ }^{\mathbb{1} 2}$, 罗振华 ${ }^{\mathbb{1} 2}$, 李春林 ${ }^{\mathbb{1} 2}$, 李春旺 ${ }^{\mathbb{1}}$, 蒋志刚 ${ }^{\mathbb{1} 2^{*}}$ \\ (1) 中国科学院动物研究所动物生态与保护生物学院重点实验室, 北京 100101 ; \\ (2) 中国科学院大学, 北京 100049 \\ * 联系人, E-mail: jiangzg@ioz.ac.cn
}

2012-05-15 收稿, 2012-07-31 接受

中国科学院知识创新工程(CXTDS2005-4, KSCX2-EW-J-2)、国家科技支撑计划(2008BAC39B04)和国家自然科学基金(30670267, 30430120) 资助

\begin{abstract}
摘要 一些研究者认为狗獾是蚯蚓专食者. 本文对欧亚狗獾 (Meles spp.)的食性和相关的地理 因子(纬度、海拔和坡向等)和环境因子(温度、雪被厚度、降水、初级生产力和人类影响等)之 间的关系进行了详细的分析, 检验了这一假说. 加上本研究组在青海湖地区的狗獾食性的研究 数据, 我们共采用了 19 篇文献中报道的 22 个研究地点的数据用于狗獾食性与生物地理关系分 析. 采用主成分分析法(principal component analysis, PCA)甄别了狗獾食性类别, 提取影响狗獾 食性重要作用的主成分; 应用多元回归分析揭示了食物多样性与主成分因子及生物地理因子 之间的关系. 研究发现, 狗獾食物的构成有明显的纬度梯度. 狗獾在低纬度地区主要以昆虫和 爬行动物为食, 而在高纬度地区则代之以蚯蚂为食, 狗獾不是一个蚯蚂专食者. 进一步研究表 明, 狗獾的食物多样性与纬度和人类影响强度呈显著正相关. 食物的丰富度和可获得性决定了 狗獾的采食策略. 狗獾善于利用当地的资源作为自己的主要食物. 从整个狗獾分布区来看, 狗 獾是一个机会广食者.
\end{abstract}

\section{关键词}

环境因子

采食生态学

广食者

纬度梯度

食物多样性
食肉目动物是生态系统中一类非常重要的关键 物种. 地理因子影响食肉动物食物的可获得性, 并对 其食物的种类和多样性产生影响. 例如, 在南方地区, 欧洲水獭(Lutra lutra) 的食物多样显著高于北方地 $区^{[1]}$. 其他肉食动物, 如野猫 (Felis silvestris)、貂 (Martes spp.) 和赤狐 (Vulpes vulpes) 的食物种类多样 性也呈现相似的纬度梯度 ${ }^{[2 \sim 4]}$. 在低纬度地区, 野猫 主要捕食野兔, 而在高纬度地区则主要以啮齿目动 物为食物 ${ }^{[2]}$. 松貂在北方地区主要以哺乳动物和鸟类 为食, 而在南方地区则更多地采食植物性食物 ${ }^{[3]}$. 赤 狐在南方地区主要以兔形目和无脊椎动物为食, 在 北方地区则以小形哺乳类和植物果实为主 ${ }^{[4]}$. 另外海 拔对于赤狐的食物多样性也有影响. 在低海拔地区, 赤狐更多地捕食兔形目动物而在高海拔地区它们以
小型哺乳类动物为食 ${ }^{[4]}$. 此外, 对食肉动物采食生态 学研究发现, 环境因子对动物的食物多样性也起至 关重要作用. 例如, 温度对于貂类的食物多样性有明 显影响 ${ }^{[2]}$, 而降水量则影响小棕蝠(Myotis lucifugus) 的食物丰富度 ${ }^{[5]}$. 棕熊(Ursus arctos) 对脊椎动物的捕 食随着雪被覆盖时间和厚度的增加而增加, 而当温 度、降水和初级生产力上升时, 棕熊捕食的无脊椎动 物量增加 ${ }^{[6]}$. 总之, 研究动物捕食习性和生物地理因 子之间的关系能够使我们深人了解食肉动物生态习 性和生活史策略, 同时也有利于我们对处于濒危的 食肉目动物提出更好的保护策略 ${ }^{[7]}$.

狗獾广泛地分布于欧亚大陆(图 1). 一些研究者 把所有狗獾作为一个独立的种 ${ }^{[8,9]}$, 而一些研究者则 把狗獾分为 3 个独立的种：欧洲獾 (M. meles)、亚洲獾 2013, 58: 2225-2261, doi: 10.1007/s11434-012-5650-9 


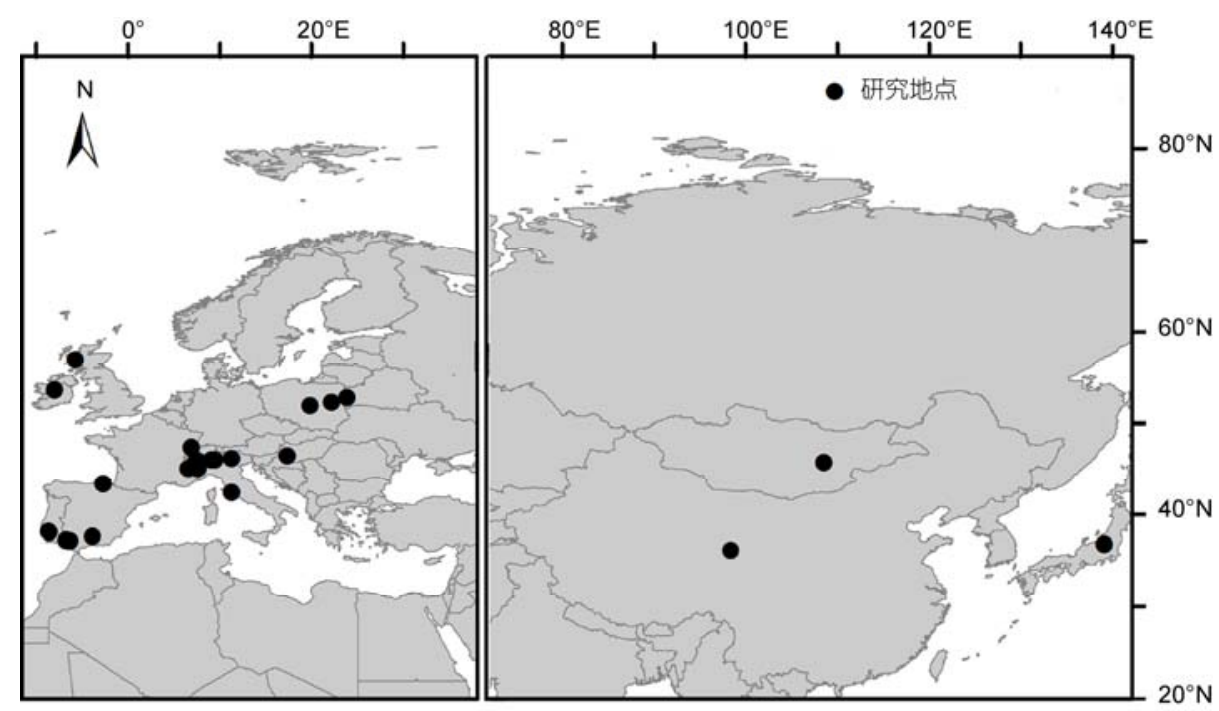

图 1 狗獾食性研究地点在欧亚大陆的分布

(M. leucurus) 和日本獾 $(M \text {. anakuma })^{[10]}$. 本研究把狗 獾看作是一个独立的种. 人们在狗獾分布范围内对 其采食生态学已经开展大量研究, 如来自英国 ${ }^{[11]}$ 、意 大利 ${ }^{[1220]}$ 、匈牙利 ${ }^{[21]}$ 、爱尔兰 ${ }^{[22]}$ 、波兰 ${ }^{[23]}$ 、日本 ${ }^{[24]}$ 、

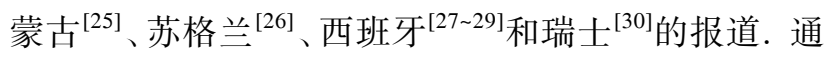
过研究狗獾食性, 一些研究认为狗獾是一种蚯蚓专 食者 ${ }^{[26,31]}$, 而另外一些研究却证明狗獾是一个广食 者 ${ }^{[19,27,29]}$, 主要根据食物的可获得性程度来利用资源. 狗獾选择食物时表现出了很大的弹性: 野兔、昆虫和 果实均是狗獾的潜在性食物资源, 所以说狗獾是一 个机会主义捕食者.

Goszczynski 等人 ${ }^{[23]}$ 研究了地理因子, 如纬度及 栖息地类型对于欧洲狗獾食性的影响, 揭示了狗獾 主要以蝔蚓和果实为食, 狗獾的食物组成具有纬度 梯度性. Roper 和 Lups ${ }^{[32]}$ 对前苏联狗獾的食性研究进 行了综述, 认为狗獾是一个广食者. 当容易获得动物 性食物时, 狗獾会优先利用动物性食物. 但是, 到目 前为止, 对于狗獾食物构成与环境因子如温度、降水 初级生产力和雪被等之间关系的研究尚未见报道. 因此, 我们检索了有关狗獾食性研究的文献, 对狗獾 食性构成进行了分类, 并评价了环境因子对狗獾食 性的影响.

本研究检验了狗獾是具有机会捕食主义行为的 广食者这一假设, 并着重研究了下面的内容: (i ) 狗 獾的主要食物种类与地理因子(纬度和海拔)之间的 关系; (ii ) 从生物地理方面解释了狗獾的捕食模式; (iii) 对狗獾是广食者还是专食者这一对立的观点进 了讨论.

\section{1 材料与方法}

（i ）狗獾食性研究文献检索. 对所有可获得的 数据库进行了文献检索, 选择了其中符合以下标准 的文献作为进一步分析使用: (1) 狗獾食性数据包括 狗獾一整年的活动期中的食性数据; (2) 食性数据样 本大于 60 ; (3) 文献中提供了足够的信息以便我们计 算每种食物类型的相对频次; (4) 提供了研究地点足 够的信息, 以便我们能够从地图上找到相关的地理 信息; (5) 主要采用胃内容物分析或粪便样品分析食 性. Roper 和 Lups ${ }^{[32]}$ 综述中的文献因为不符合上述标 准而被排除在分析之外. 加上我们在青藏高原青海 湖盆地对狗獾食性的研究, 总计有 19 篇文献 22 个地 点狗獾的食性研究数据用于本研究分析. 我们通过 粪样分析法获得了青海湖地区狗獾食物构成成分: 粪样用水完全浸泡后, 用 5 和 $1 \mathrm{~mm}$ 孔目的篮子过篮, 然后对留在篮子中的未消化的残余物进行辨认分类. 在青海湖盆地区, 狗獾主要捕食昆虫, 其出现频次和 相对频次分别是 $100 \%$ 和 $68.45 \%$. 其他的食物, 如高 原鼠兔 (Ochotona curzoniae)、鸟类、青海沙蜥 (Phrynocephalus vlangalii) 和麻黄为补充食物 (青海湖 地区狗獾的详细食物构成见附录 1).

我们用食物出现的相对频次来表示狗獾的食性. 狗獾的食物构成分为 9 类: 蝶蚓、鸟、哺乳类、昆虫、 
两栖类、爬行类、果实、软体动物和其他 (植物的叶、 根和垃圾等未消化的碎片). 用 Shannon-Wiener 指数 $\left(H^{\prime}\right)$ 来表示狗獾的食物多样性:

$$
H^{\prime}=-\sum P_{i} \times \log \left(P_{i}\right),
$$

其中, $P_{i}$ 表示给定食物种类 $i$ 的相对频次.

(ii) 生物地理因子. 每个研究地点的生物地理 因子包括温度、降水量、雪被厚度、归一化植被指数、 海拔、坡向、纬度和经度等. 从 1955 2000 年的世界 气候数据库(http://www.worldclim.org/current)中提取 了年平均温度 $\left(T_{\text {mean }}\right)$ 、年平均降水量 $\left(P_{\text {mean }}\right)$ 、暖季平 均温度 $\left(T_{\mathrm{warm}}\right)$ 、冷季平均温度 $\left(T_{\text {cold }}\right)$ 、旱季降水量 $\left(P_{\mathrm{dry}}\right)$ 和雨季降水量 $\left(P_{\mathrm{wet}}\right)$ 数据. 我们采用 1980 2000 年的 年平均归一化植被指数作为生产力因子. 年平均归 一化植被指数的数据来自于 GIMMS 数据库, 其空间 分辨率是 $1 \mathrm{~km} \times 1 \mathrm{~km}$ (http://glcf.umiacs.umd.edu/ data/gimms/). 我们从全球生态系统数据库中提取了 研究地点雪被的厚度 (http://www.ngdc.noaa.gov). 有 关研究地点地形的数据主要来自于 CGIAR- CSI 数据 库(http://srtm.sci.cgiar.org). 我们从中提取海拔、经纬 度和坡向等因子.人类影响以人类影响指数(human influence index, HII)表示 ${ }^{[33]}$. 人类影响指数从 8 个方 面评介人类活动对生态系统的影响, 分别是人口密 度评分 $(0 \sim 10)$ 、铁路评分 $(8,4$ 和 0$)$ 、干线道路评分 $(8$, 4 和 0$)$ 、通航河流评分 (4 和 0$)$ 、海岸线评分 (4 和 0$)$ 、 夜晚持续灯火评分 $(0,3,6$ 和 10)、城市评分 $(10$ 和 0$)$ 以及土地覆盖评分 $(10,8,3$ 和 0$)$. HII 为这 8 个人类影 响因子的得分总和，取值范围在 0 64 之间, 0 表示无 人类活动影响, 64 则表示人类活动影响最大. HII 数 据来自于 SEDAC 数据库, 其空间分辨率为 $30 \mathrm{~s}$ (http://sedac.ciesin.columbia.edu/wildareas/).

(iii) 数据分析. 我们采用 K-S 检验对所有获得 的数据进行了正态性检验, 如不符合正态分布进行 转换使之符合正态分布 ${ }^{[34]}$. 我们采用主成分分析 (principal component analysis, PCA)对狗獾的食物构 成进行分析, 以达到消除食物各种类之间的共线性 关系的目的. 我们采用 Pearson 相关对主成分因子和 纬度及海拔进行相关分析. 在线性回归中, 主成分因 子作为应变量, 纬度和海拔作为自变量进行回归分 析. 同样, 我们对食物多样性指数和纬度, 海拔及坡 向之间的关系进行了回归分析. 另外, 我们还对食物 构成和环境因子对食物多样性指数的影响进行了回
归分析. 所有统计分析均采用 SPSS 16.0, 统计显著 水平为 0.05 .

\section{2 结果}

\section{1 狗獾食物多样性构成}

从所有狗獾食性研究来看, 昆虫和果实是狗獾 采食的主要食物种类(表 1); 而蛏蚓并非狗獾的主要 食物. 对狗獾食物构成进行了 PCA 分析, 共产生了 4 个特征值大于 1 的主成分因子, 解释了超过 $82 \%$ 的方 差 (表 2). 第一主成分特征值为 2.54 , 其贡献率为 $28.23 \%$, 其中, 蚯蚓和其他食物种类分值分别是 0.70 和 0.59 , 而昆虫和爬行类则表现为较高的负向量, 分 别为 -0.92 和 -0.81 . 第二主成分的特征值为 1.91 , 贡 献率为 $21.23 \%$, 其中, 哺乳类和鸟类分值较大, 分别 为 0.93 和 0.73 , 而蛏蚓表现为较高的负向量, 为 -0.51 . 第三主成分的特征值为 1.56 , 贡献率为 $17.29 \%$, 其中, 两栖类分值最大, 为 0.88 , 而果实则 表现较高的负向量, 为 -0.79 . 第四主成分特征值为 1.41 , 其贡献率为 $15.63 \%$, 其中, 鸟类和软体动物分 值最大, 分别是 0.53 和 0.94. 多元回归分析表明, 纬 度与第一主成分成呈显著正相关 $($ 第一主成分 $=$ $-4.078+0.090 \times$ 纬度, $R^{2}=0.269, F_{21}=7.352, P=$ 0.01 , 图 2(a)). 所有主成分与海拔没有显著相关.

各主成分与环境因子的多元回归分析表明，主 成分中仅第一主成分与环境因子中的旱季降水和人 类影响指数显著相关 $($ 第一主成分 $=-1.820+0.006 \times$ $P_{\mathrm{dry}}+0.045 \times \mathrm{HII}\left(R^{2}=0.500, F_{21}=9.488, P=0.001\right.$, 图 2(b)和 $(\mathrm{c})))$. 其他主成分与环境因子相关不显著 $(P$ $>0.05)$.

\section{2 影响狗獾食物多样性的因素分析}

狗獾食物多样性与食物种类之间的多元回分析 表明: 食物多样性指数与昆虫和蝶蚓的相对频次呈 显著负相关 $\left(R^{2}=0.702, F_{21}=22.362, P=0.000\right.$; 图 3). 食物多样性指与地理因子中的纬度呈显著正相关 $\left(R^{2}\right.$ $=0.199, F_{21}=4.97, P=0.037$; 图 4). 此外, 食物多样 性指还与环境因子中的人类影响指数呈正相关 $\left(R^{2}=\right.$ $0.204, F_{21}=5.118, P=0.035$; 图 4).

\section{3 讨论}

狗獾对于食物的选择具有很高的弹性, 植物、环 
表 1 狗獾食性研究中各种食物相对频次构成

\begin{tabular}{|c|c|c|c|c|c|c|c|c|c|c|c|c|c|}
\hline 编号 & 作者 & 地点 & $\begin{array}{l}\text { 样本 } \\
\text { 容量 }\end{array}$ & $\begin{array}{c}\text { 蚳蚂 } \\
(\%)\end{array}$ & $\begin{array}{c}\text { 哺乳类 } \\
(\%)\end{array}$ & $\begin{array}{l}\text { 鸟类 } \\
(\%)\end{array}$ & $\begin{array}{c}\text { 昆虫 } \\
(\%)\end{array}$ & $\begin{array}{c}\text { 两栖类 } \\
(\%)\end{array}$ & $\begin{array}{c}\text { 爬行类 } \\
(\%)\end{array}$ & $\begin{array}{c}\text { 果实 } \\
(\%)\end{array}$ & $\begin{array}{c}\text { 软体动物 } \\
(\%)\end{array}$ & $\begin{array}{l}\text { 其他 } \\
(\%)\end{array}$ & $H^{\prime}$ \\
\hline 1 & Balestrieri (2004) & $45^{\circ} 2^{\prime} \mathrm{N}, 7^{\circ} 43^{\prime} \mathrm{E}$ & 199 & 44.93 & 6.58 & 3.01 & 18.36 & 11.24 & 0.30 & 10.68 & 4.66 & 0.24 & 1.61 \\
\hline 2 & Balestrieri (2009) & $45^{\circ} 53^{\prime} \mathrm{N}, 7^{\circ} 37^{\prime} \mathrm{E}$ & 222 & 44.58 & 2.22 & 0.25 & 20.20 & 11.33 & 0.49 & 20.94 & 0 & 0 & 1.38 \\
\hline 3 & Boesi (2002) & $46^{\circ} 01^{\prime} \mathrm{N}, 9^{\circ} 09^{\prime} \mathrm{E}$ & 118 & 8.04 & 6.92 & 1.79 & 47.99 & 0.67 & 0.22 & 34.38 & 0 & 0 & 1.23 \\
\hline 4 & Cleary (2009) & $53^{\circ} 31^{\prime} \mathrm{N}, 7^{\circ} 59^{\prime} \mathrm{W}$ & 686 & 43.12 & 1.17 & 0.59 & 41.95 & 5.7 & 0 & 6.29 & 1.17 & 0 & 1.20 \\
\hline 5 & Fedriani (1998) & $37^{\circ} 10^{\prime} \mathrm{N}, 6^{\circ} 23^{\prime} \mathrm{W}$ & 145 & 0 & 30.21 & 1.37 & 42.64 & 7.64 & 5.63 & 12.52 & 0 & 0 & 1.40 \\
\hline 6 & Del Bove (2001) & $42^{\circ} 23^{\prime} \mathrm{N}, 11^{\circ} 24^{\prime} \mathrm{E}$ & 69 & 0 & 15.50 & 7.50 & 52.50 & 0 & 0.50 & 14.50 & 9.50 & 0 & 1.35 \\
\hline 7 & Fischer (2005) & $47^{\circ} 19^{\prime} \mathrm{N}, 6^{\circ} 58^{\prime} \mathrm{W}$ & 1436 & 28.02 & 4.48 & 0 & 19.02 & 0 & 0 & 43.03 & 0 & 5.45 & 1.33 \\
\hline 8 & Gonavalski (2000) & $52^{\circ} 43^{\prime} \mathrm{N}, 23^{\circ} 54^{\prime} \mathrm{E}$ & 141 & 24.43 & 15.66 & 0 & 17.94 & 0 & 0 & 36.75 & 0 & 5.22 & 1.46 \\
\hline 9 & Gonavalski (2000) & $52^{\circ} 13^{\prime} \mathrm{N}, 22^{\circ} 13^{\prime} \mathrm{E}$ & 281 & 31.9 & 15.19 & 0 & 27.90 & 0 & 0 & 19.76 & 0 & 5.25 & 1.48 \\
\hline 10 & Gonavalski (2000) & $51^{\circ} 48^{\prime} \mathrm{N}, 19^{\circ} 53^{\prime} \mathrm{E}$ & 66 & 24.18 & 11.12 & 1.51 & 41.28 & 13.52 & 0.37 & 4.35 & 0 & 3.67 & 1.56 \\
\hline 11 & Kaneko (2006) & $36^{\circ} 45^{\prime} \mathrm{N}, 139^{\circ} 15^{\prime} \mathrm{E}$ & 82 & 33.55 & 1.94 & 4.52 & 21.29 & 0.65 & 0 & 35.48 & 2.58 & 0 & 1.41 \\
\hline 12 & Kruuk (1981) & $56^{\circ} 53^{\prime} \mathrm{N}, 5^{\circ} 49^{\prime} \mathrm{W}$ & 2159 & 26.37 & 6.49 & 2.41 & 39.98 & 1.49 & 0 & 19.69 & 0 & 3.57 & 1.49 \\
\hline 13 & Lanszki (2004) & $46^{\circ} 24^{\prime} \mathrm{N}, 17^{\circ} 27^{\prime} \mathrm{E}$ & 156 & 17.55 & 5.16 & 1.21 & 44.97 & 23.39 & 1.03 & 6.33 & 0 & 0.36 & 1.45 \\
\hline 14 & Lucherini (1995) & $45^{\circ} 04^{\prime} \mathrm{N}, 6^{\circ} 39^{\prime} \mathrm{E}$ & 76 & 22.44 & 11.21 & 0.93 & 59.82 & 0 & 0 & 5.60 & 0 & 0 & 1.09 \\
\hline 15 & Marassi (2002) & $46^{\circ} 01^{\prime} \mathrm{N}, 9^{\circ} 30^{\prime} \mathrm{E}$ & 147 & 20.04 & 6.41 & 1.45 & 29.06 & 0 & 0 & 41.28 & 1.15 & 0.61 & 1.37 \\
\hline 16 & Martin (1995) & $37^{\circ} 07^{\prime} \mathrm{N}, 6^{\circ} 44^{\prime} \mathrm{W}$ & 530 & 0 & 26.82 & 1.42 & 54.36 & 6.99 & 7.13 & 3.28 & 0 & 0 & 1.23 \\
\hline 17 & Murdoch (2010) & $45^{\circ} 43^{\prime} \mathrm{N}, 108^{\circ} 39^{\prime} \mathrm{E}$ & 116 & 0 & 14.77 & 3.98 & 63.64 & 0 & 12.5 & 5.11 & 0 & 0 & 1.11 \\
\hline 18 & Prignioni (2008) & $46^{\circ} 07^{\prime} \mathrm{N}, 11^{\circ} 20^{\prime} \mathrm{E}$ & 230 & 11.90 & 4.84 & 2.22 & 27.33 & 2.56 & 0 & 51.14 & 0 & 0 & 1.28 \\
\hline 19 & Remonti (2007) & $45^{\circ} 38^{\prime} \mathrm{N}, 7^{\circ} 20^{\prime} \mathrm{E}$ & 228 & 0 & 41.58 & 11.70 & 14.98 & 0 & 0 & 26.60 & 0 & 5.14 & 1.41 \\
\hline 20 & Zabala (2002) & $43^{\circ} 23^{\prime} \mathrm{N}, 2^{\circ} 40^{\prime} \mathrm{W}$ & 80 & 29.68 & 3.26 & 0 & 37.21 & 0 & 0 & 25.58 & 0 & 4.23 & 1.32 \\
\hline 21 & Rosalino (2005) & $38^{\circ} 06^{\prime} \mathrm{N}, 8^{\circ} 36^{\prime} \mathrm{W}$ & 282 & 0 & 1.36 & 0.14 & 66.32 & 0.47 & 0.3 & 31.40 & 0 & 0 & 0.75 \\
\hline 22 & 本研究(2010) & $36^{\circ} 40^{\prime} \mathrm{N}, 100^{\circ} 48^{\prime} \mathrm{E}$ & 162 & 0 & 3.34 & 5.41 & 68.45 & 0 & 13.54 & 8.87 & 0.4 & 0 & 1.04 \\
\hline
\end{tabular}

表 2 狗獾食性主成分析及主成分与纬度和海拔相关分析 ${ }^{\text {a) }}$

\begin{tabular}{|c|c|c|c|c|}
\hline & \multicolumn{4}{|c|}{ 主成分 } \\
\hline & 1 & 2 & 3 & 4 \\
\hline 蚯蚓 & 0.70 & -0.53 & 0.26 & -0.02 \\
\hline 哺乳类 & -0.03 & 0.93 & 0.08 & -0.10 \\
\hline 鸟类 & -0.09 & 0.73 & -0.13 & 0.53 \\
\hline 昆虫 & -0.92 & -0.12 & 0.08 & 0.03 \\
\hline 两栖类 & 0.12 & -0.14 & $0.88^{*}$ & -0.02 \\
\hline 爬行类 & -0.81 & 0.23 & 0.11 & -0.12 \\
\hline 果实 & 0.43 & -0.16 & -0.79 & -0.07 \\
\hline 软体动物 & 0.08 & 0.05 & 0.02 & 0.94 \\
\hline 其他 & 0.59 & 0.37 & -0.21 & -0.47 \\
\hline 特征值 & 2.54 & 1.91 & 1.56 & 1.41 \\
\hline 解释方差(\%) & 28.23 & 21.23 & 17.29 & 15.63 \\
\hline$R_{\text {latitude }}$ & $0.52^{*}$ & -0.09 & 0.10 & -0.26 \\
\hline$R_{\text {elevation }}$ & -0.29 & 0.03 & -0.26 & 0.01 \\
\hline
\end{tabular}



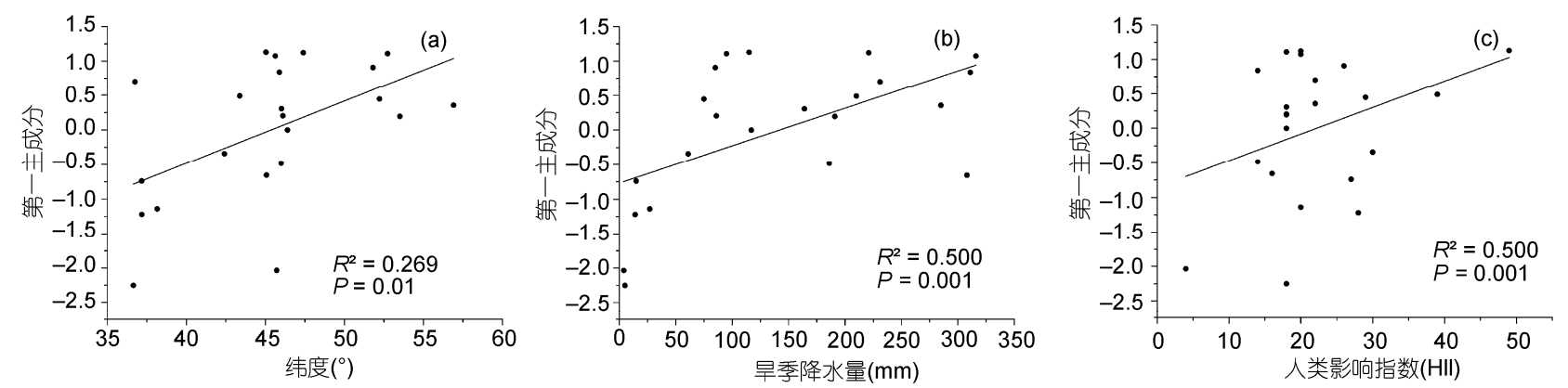

图 2 狗獾食性 PCA 分析中的第一主成分与纬度 $(a)$ 、旱季降水量(b)和人类影响指数(c)之间的关系
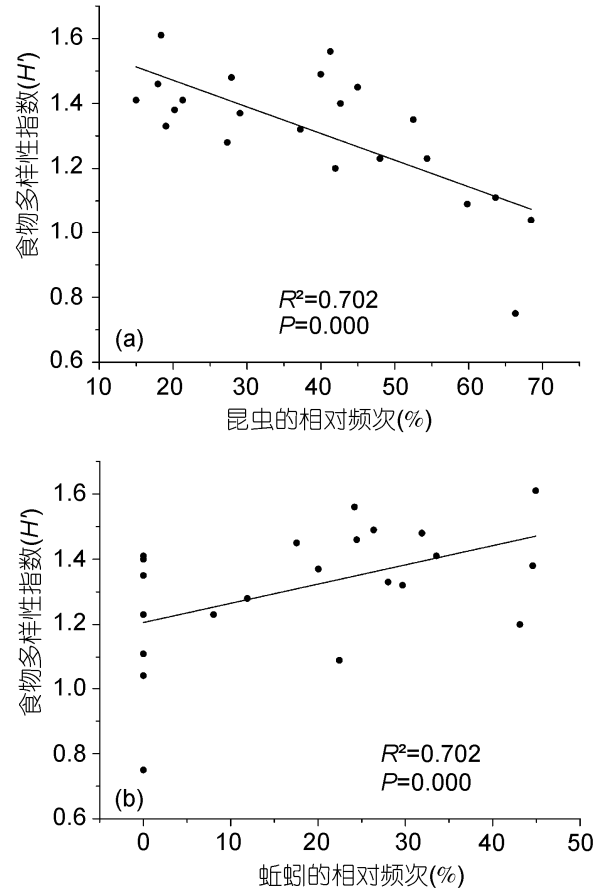

图 3 狗獾食物多样性指数与昆虫(a)和蝶蚂(b)相对频次之间 的关系

节动物、软体动物、昆虫、两栖爬行类、鸟类和哺乳 类均是狗獾潜在的食物. 在英国, 狗獾主要捕食蚯 蚂 ${ }^{[11]}$; 而在其他地区，昆虫、哺乳类和果实是狗獾的 主要食物 ${ }^{[14,23 ~ 25]}$. 地理环境差异导致食物资源可获 得性的差异, 也许部分地解释了狗獾食性变化的 原因 ${ }^{[35]}$. 在干旱的低纬度地区, 环境中昆虫和爬行 动物食物量丰富一些, 而在高纬度湿润地区, 虾蚓 量丰富. 所以, 狗獾在低纬度干旱地区主要捕食昆虫 和爬行类，而在高纬度湿润地区狗獾捕食蚯蚓的 频次增加 ${ }^{[14,21,26]}$. 类似的情况也在其他食肉目动物中 出现. 例如, 野猫在北方主要以鼠类为食而在南方地 区则以野兔为食 ${ }^{[2]}$. 赤狐也表现与野猫相同的食性.
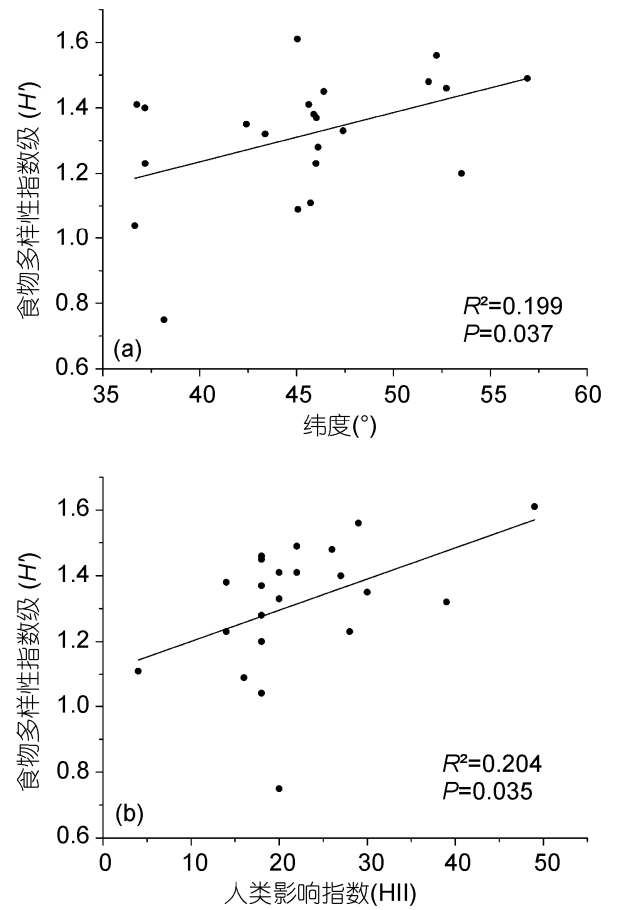

图 4 狗獾食物多样性指数与纬度 (a)和人类影响指数 (b) 之间的关系

当果实量丰富时, 松貂则从捕食哺乳类转为以果实为 食 ${ }^{[3]}$.

狗獾只在少数几个地点以螘蚓为主要食物, 所以 狗獾不应该看做是蝶蚓专食者. 根据 Hanski 等人 ${ }^{[36]}$ 对于广食者的定义, 狗獾从其分布范围来看应该视为 一个广食者. 狗獾善于利用当地食物资源. 在一些地 点, 爬行类、哺乳类、昆虫和果实在狗獾食物中的比 例异常的高, 因为这些动物在当地的生物量非常丰富. 例如, 狗獾在葡萄牙南部的柇树林中主要采食果实 (主要是橄榄、梨和无花果) ${ }^{[27]}$. 在蒙古干旱区, 狗獾则 主要捕食昆虫 ${ }^{[25]}$. 而在西班牙的 Doñana 国家公园则 以野兔、昆虫和果实为主要食物 ${ }^{[28]}$. 
从广阔的地理分布范围内来看, 野生动物的食 物多样性通常受到纬度的影响. 很多研究证实食物 多样性会随着纬度的上升而下降. 在北半球, 乌灰䍃 (Circus pygargus)的食物多样性由南向北逐步下降 ${ }^{[37]}$. 野猫也表现了类似的趋势. 然而, 狗獾的食物多样性 却表现出了相反的趋势. Goszcynski 等人 ${ }^{[23]}$ 综述欧洲 地区的狗獾食性时同样发现了狗獾的食物多样性在 中纬度地度最高. Zhou 等人 ${ }^{[3]}$ 认为松貂在南方低纬度 地区具有更高的食物多样和生态位宽度, 因为昆虫 不是松貂的主要食物. 对于狗獾来说, 在南方干旱地 区，昆虫恰恰是狗獾的主要食物. 所以，不难理解狗 獾在南方低纬度地区的食物多样性比北方高纬度地 区低.

初级生产力通常和物种多样性和食物多样性相 关. 有研究表明, 在初级生产力高的地方, 鸟的种类 也会随之增加 ${ }^{[38]}$. 初级生产力也是一个能够较好地 预测食草动物和食肉动物分布的因素. 蒙原羚 (Procapra gutturosa) 比较喜欢选择初级生产力中等 的地方作为自己的生境 ${ }^{[39]}$. 在初级生产力低的地区, 初级生产力会对狍的体重产生显著影响 ${ }^{[00]}$. 棕熊对 于果实的取食与初级生产力呈显著正相关 ${ }^{[6]}$. 但是, 我们的研究表明, 初级生产力对于狗獾的食物构成 不产生显著影响. Zhou 等人 ${ }^{[3]}$ 认为松貂仅仅利用很少
种类果树的果实，而植被初级生产力包括所有植物 的初级生产力, 动物利用的果树的初级生产力只是 其中一小部分, 所以初级生产力并不能真实反映松 貂的食物构成. 狗獾采食动物性食物为主, 采食果实 只是补充食物, 并且采食果实具有季节性. 这也解释 了为什么植被初级生产力对狗獾的食性没有显著相 关的原因.

我们的结果揭示了狗獾的食物多样性与人类活 动强度呈正相关. 人类活动导致野生动物栖息地破 碎化 ${ }^{[41]}$. 研究表明, 物种丰富度会随人类活动强度 的上升而下降 ${ }^{[22,43]}$, 而物种多样性和丰富度在不同 生境变化显著, 尤其是昆虫的多样性和丰富度 ${ }^{[44]}$. 狗獾的采食生境通常包含多种类型. 在活动期, 狗獾 捕食时会在几种不同生境类型中捕食. 这也许是狗 獾在人类干扰强度高的地点具有高的食物多样性的 原因. 另一原因是在靠近人类的地方，狗獾有机会利 用人类栽种的水果. 动物对果实的利用会增加动物 的食物多样性, 这一点在松貂上已得到证实 ${ }^{[3]}$.

总之, 分布在欧亚大陆的狗獾食谱包括脊椎动 物和无脊椎动物, 甚至包括植物性资源. 狗獾的食物 多样性变化呈现出纬度梯度. 狗獾善于开发利用当 地的食物资源, 甚至能在人类活动的地点寻找食物. 狗獾是一个采用机会主义捕食策略的广食者.

\section{参考文献}

1 Clavero M, Prenda J, Delibes M. Trophic diversity of the otter (Lutra lutra L.) in temperate and Mediterranean freshwater habitats. J Biogeogr, 2003, 30: 761-769

2 Lozano J, Moleon M, Virgos E. Biogeographical patterns in the diet of the wildcat, Felis silvestris Schreber, in Eurasia: Factors affecting the trophic diversity. J Biogeogr, 2006, 33: 1076-1085

3 Zhou Y B, Newman C, Xu W T, et al. Biogeographical variation in the diet of Holarctic martens (genus Martes, Mammalia: Carnivora: Mustelidae): Adaptive foraging in generalists. J Biogeogr, 2011, 38: 137-147

4 Díaz-Ruiz F, Delibes-Mateos M, García-Moreno J L, et al. Biogeographical patterns in the diet of an opportunistic predator: The red fox Vulpes vulpes in the Iberian Peninsula. Mammal Rev, 2011, doi: 10.1111/j.1365-2907.2011.00206.x

5 Grindal S D, Collard T S, Brigham R M, et al. The influence of precipitation on reproduction by myotis bats in British-Columbia. Am Midl Nat, 1992, 128: 339-344

6 Bojarska K, Selva N. Spatial patterns in brown bear Ursus arctos diet: The role of geographical and environmental factors. Mammal Rev, 2011, 4: 120-142

7 Daan S, Tinbergen J M. Adaptation of life histories. In: Krebs J R, Davies N B, eds. Behavioural Ecoloy: An Evolutionary Approach. Oxford UK: Wiley-Blackwell, 1997

8 Nowak R M. Walker's Mammals of the World. 6th ed. Baltimore: Johns Hopkins University Press, 1999

9 冯祚建. 西藏哺乳类. 北京: 科学出版社, 1986. 161-162

10 Abramov A. Variation of the baculum structure of the Palaearctic badger (Carnivora, Mustelidae, Meles). Russian J Theriol, 2002, 1: $57-60$

11 Dasilva J, Woodroffe R, Macdonald D W. Habitat, food availability and group territoriality in the European Badger, Meles meles Oecologia, 1993, 95: 558-564 
12 Zabala J, Garin I, Zuberogoitia I, et al. Habitat selection and diet of badgers (Meles meles) in Biscay (northern Iberian Peninsula). Ital J Zool, 2002, 69: 233-238

13 Lucherini M, Crema G. Seasonal variation in the food habits of badgers in an Alpine valley. Hystrix, 1995, 7: 165-171

14 Balestrieri A, Remonti L, Prigioni C. Diet of the Eurasian badger (Meles meles) in an agricultural riverine habitat (NW Italy). Hystrix, 2004, 15: 3-12

15 Prigioni C, Balestrieri A, Remonti L, et al. Differential use of food and habitat by sympatric carnivores in the eastern Italian Alps. Ital J Zool, 2008, 75: 173-184

16 Balestrieri A, Remonti L, Prigioni C. Exploitation of food resources by the Eurasian badger (Meles meles) at the altitudinal limit of its Alpine range (NW Italy). Zool Sci, 2009, 26: 821-827

17 Remonti L, Balestrieri A, Prigioni C. Role of fruits in the diet of small mustelids (Mustela sp.) from the western Italian Alps. Eur J Wildl Res, 2007, 53: 35-39

18 Marassi M, Biancardi C. Diet of the Eurasian badger (Meles meles) in an area of the Italian Prealps. Hystrix, 2002, 13: 19-28

19 Boesi R, Biancardi C M. Diet of the Eurasian badger Meles meles (Linnaeus, 1758) in the natural reserve of Lago di Piano, northern Italy. Mamm Biol, 2002, 67: 120-125

20 Del Bove E, Isotti R. The European badger (Meles meles) diet in a Mediterranean area. Hystrix, 2001, 12: 19-25

21 Lanszki J. Diet of badgers living in a deciduous forest in Hungary. Mamm Biol, 2004, 69: 354-358

22 Cleary G, Corner L, O'Keeffe J, et al. The diet of the badger Meles meles in the Republic of Ireland. Mamm Biol, 2009, 74: 438-447

23 Goszczynski J, Jedrzejewska B, Jedrzejewski W. Diet composition of badgers (Meles meles) in a pristine forest and rural habitats of Poland compared to other European populations. J Zool, 2000, 250: 495-505

24 Kaneko Y, Maruyama N, Macdonald D W. Food habits and habitat selection of suburban badgers (Meles meles) in Japan. J Zool, 2006, 270: 78-89

25 Murdoch J D, Buyandelger S. An account of badger diet in an arid steppe region of Mongolia. J Arid Environ, 2010, 74: 1348-1350

26 Kruuk H, Parish T. Feeding specialization of the European Badger Meles meles in Scotland. J Anim Ecol, 1981, 50: 773-788

27 Rosalino L M, Loureiro F, MacDonald D W, et al. Dietary shifts of the badger (Meles meles) in Mediterranean woodlands: An opportunistic forager with seasonal specialisms. Mamm Biol, 2005, 70: 12-23

28 Fedriani J M, Ferreras P, Delibes M. Dietary response of the Eurasian badger, Meles meles, to a decline of its main prey in the Donana National Park. J Zool, 1998, 245: 214-218

29 Martin R, Rodriguez A, Delibes M. Local feeding specialization by Badgers (Meles meles) in a Mediterranean environment. Oecologia, 1995, 101: 45-50

30 Fischer C, Ferrari N, Weber J M. Exploitation of food resources by badgers (Meles meles) in the Swiss Jura mountains. J Zool, 2005, 266: $121-131$

31 Virgos E, Llorente M, Cortes Y. Geographical variation in genet (Genetta genetta L.) diet: A literature review. Mammal Rev, 1999, 29: $117-126$

32 Roper T, Lups P. Diet of badgers (Meles meles) in central Switzerland: An analysis of stomach contents. Mamm Biol, 1995, 60: 9-19

33 Sanderson E W, Jaiteh M, Levy M A, et al. The human footprint and the last of the wild. Bioscience, 2002, 52: 891-904

34 Underwood A J. Experiments in Ecology. London: Cambridge University Press, 1996

35 Chapman R F. The Insects: Structure and Function. London: Cambridge University Press, 1998

36 Hanski I, Hansson L, Henttonen H. Specialist predators, generalist predators, and the microtine rodent cycle. J Anim Ecol, 1991, 60: 353-367

37 Terraube J, Arroyo B. Factors influencing diet variation in a generalist predator across its range distribution. Biodivers Conserv, 2011, 20: 2111-2131

38 Lee P F, Ding T S, Hsu F H, et al. Breeding bird species richness in Taiwan: Distribution on gradients of elevation, primary productivity and urbanization. J Biogeogr, 2004, 31: 307-314

39 Mueller T, Olson K A, Fuller T K, et al. In search of forage: Predicting dynamic habitats of Mongolian gazelles using satellite-based estimates of vegetation productivity. J Appl Ecol, 2008, 45: 649-658

40 Pettorelli N, Gaillard J M, Mysterud A, et al. Using a proxy of plant productivity (NDVI) to find key periods for animal performance: The case of roe deer. Oikos, 2006, 112: 565-572

41 Vitousek P M, Mooney H A, Lubchenco J, et al. Human domination of earth's ecosystems. Science, 1997, 277: 494-499

42 Laliberte A S. Ripple W J. Range contractions of North American carnivores and ungulates. Bioscience, 2004, 54: 123-138

43 Lopes M A, Ferrari S F. Effects of human colonization on the abundance and diversity of mammals in eastern Brazilian Amazonia. Conserv Biol, 2000, 14: 1658-1665 
44 Janzen D H, Schoener T W. Differences in insect abundance and diversity between wetter and drier sites during a tropical dry season. Ecology, 1968, 49: 96-110

\section{补充材料}

\section{附录 1 狗獾食性鉴定方法}

本文以上补充材料见网络版 csb.scichina.com. 补充材料为作者提供的原始数据, 作者对其学术质量和内容负责.

- 动 态 $\cdot$

\section{研究发现 H7N9 流感病毒适应感染哺乳动物的新突变}

最近的一项研究发现, 某些 H7N9 流感病毒的基因序 列中含有一个新型的突变, 可能在这种新型流感病毒的感 染和发病过程中发挥一定的作用.

题名为“新型 H7N9 禽流感病毒感染与环境流感病毒 的关联和病毒对宿主的适应”的研究论文发表于《中国科学: 生命科学》英文版 2013 年第 6 期, 从流行病学、临床医学 和生物信息学等角度探讨了新型 H7N9 禽流感病毒的特征, 由浙江省杭州市疾控中心潘劲草教授和中国科学院微生物 所刘军副研究员担任通讯作者撰写, 中国疾病预防控制中 心高福副主任指导了该研究.

2013 年 4 月 1 日, 来自杭州市疾控中心微生物实验室 的研究者利用基因组测序技术独立鉴定了浙江省首个 H7N9 禽流感感染病例. 4 月 5 日, 又在与浙江省第二例病 例有关联的活禽市场标本中检出 H7N9 禽流感病毒核酸, 为揭示传染源提供了可靠证据. 该实验室研究者在承担了 杭州市 H7N9 监测病例和外环境标本的大量检测工作外, 还与中国科学院微生物所等多家科研机构开展了有关 H7N9 禽流感病毒序列特征、外环境病毒遡源、病毒关键 位点突变与宿主适应关系等方面的研究.

这次《中国科学: 生命科学》发表的论文报道了浙江 省杭州市的 3 个较早的 H7N9 感染病例, 有 2 例具有明确 的活禽市场接触史, 而且在其中 1 例病人发病前去过的活 禽市场检测到了与病人上呼吸道样本中高度同源的 $\mathrm{H} 7 \mathrm{~N} 9$ 病毒, 对于人感染 H7N9 流感病毒的来源和传播途径的阐 明具有一定的意义.
研究者将这 3 例病人以及相关环境样本当中检测到的 H7N9 流感病毒的基因与既往报道的 H7N9 流感病毒基因 序列进行了比对, 发现这 4 株流感病毒与其他报道的 H7N9 流感病毒在遗传学上属于同一分支, 但是进一步分析发现, 仅第二个病人样本中检测到的病毒与其去过的活禽市场检 测到的病毒相邻较近, 表明他们具有高度相似性, 而其他 两个病人样本中分离到的病毒与之相去较远, 表明这些病 例中分离得到的新型 H7N9 流感病毒具有多样的基因序列.

重要的是, 研究者对一例病人的样本中检测到的流感 进行了全基因组测序, 发现这株病毒与既往报道的 H7N9 流感病毒相比, 在与禽流感病毒在哺乳动物传播相关的关 键蛋白位点上有一个氨基酸的突变. 国外既往的研究表明, 实验室中将 $\mathrm{H} 5 \mathrm{~N} 1$ 禽流感病毒的血凝素蛋白的 226 位氨基 酸由谷氨酰胺突变为亮氨酸有助于病毒结合哺乳动物细胞 受体. 而在这项研究中, 研究者发现一株 H7N9 流感病毒 在该位点具有一个异亮氨酸, 这在既往报道的 H7N9 流感 病毒的基因序列中没有被发现过. 研究人员推测这一位点 的异亮氨酸可能与 226 位亮氨酸发挥同样作用, 但其作用 机制还需进一步的实验证实.

该研究发现对于阐述新型 H7N9 流感病毒跨宿主传播 机制及其发病机理具有参考价值, 此外, 对于相应的药物 设计和疫苗研发等具有重要的科学意义.

研究得到了杭州市医学重点专科卫生检验学科资助. 


\section{网络版附录 1 狗獾食性鉴定方法}

青海湖地区狗獾样品收集是我们研究组在野外工作时收集的. 采集方法主要参照 Goszczynski 所采用的方法 ${ }^{[24]}$. 样 品收集时间从 2008 年 10 月到 2010 年 10 月, 收集样品地点为青海湖湖东地区 $\left(36^{\circ} 39^{\prime} \mathrm{N}, 100^{\circ} 49^{\prime} \mathrm{E}\right)$. 在狗獾的活动期内, 我 们从典型的狗獾侧所中收集粪便样品. 其他地方的类似于狗獾粪便样品不在我们收集范围, 那些陈旧的或是腐烂的样品 同样不收集. 每周收集一次, 每次在同一则所中收集的样品作为一个样本. 样品用密封袋装好标上时间和地点, 在 $-12^{\circ} \mathrm{C}$ 中保存带回实验室. 分析前, 粪样在 $50^{\circ} \mathrm{C}$ 烘箱中烘 $48 \mathrm{~h}$ 进行烘干并称重. 然后把粪样完全浸泡于水中, 分别用孔目 $5 \mathrm{~mm}$ 和 $1 \mathrm{~mm}$ 的篮子过篮, 残液用烧杯收集, 并在显微镜下观察是否有蚯蚓的刚毛存在, 判断狗獾是否捕食蚯蚂. 留在篮子中 残余物倒人到一大的托盘中进行分类, 将残余物分为哺乳类、鸟类、两栖类、爬行类、昆虫、果实、软体动物和其他. 分 析表明青海湖地区狗獾的食物相对频次如下：哺乳类， $3.34 \%$; 鸟类, $5.41 \%$; 昆虫, $68.45 \%$; 爬行类, $13.54 \%$; 软体动物, $0.4 \%$; 和果实, $8.87 \%$. 